\title{
Meduloblastoma de alto riesgo en recaída con respuesta favorable a un análogo de somatostatina (octreótido). Reporte de un caso
}

- Carlos Eduardo Bonilla González, Diego Mauricio González Ramírez, Luisa Alejandra Galvis Gómez

Instituto Nacional de Cancerología, Bogotá, D.C.

Contacto: carlosbonillamd@gmail.com

Introducción y objetivos. El meduloblastoma cerebeloso en adultos es una enfermedad infrecuente, por lo que la mayoría de los datos del tratamiento de las recaídas se basa en series y reportes de casos de centros de referencia. Se ha identificado expresión de receptores de somatostatina en la mayoría de casos de meduloblastoma y algunas experiencias preclínicas han mostrado respuesta a los análogos de somatostatina.

Materiales y métodos. Se presenta el caso de una paciente actualmente de 20 años de edad que debutó con un meduloblastoma cerebeloso izquierdo de alto riesgo desde los 16 años, quien manifestó progresiones rápidas después de radioterapia craneospinal y dos líneas de quimioterapia.

Resultados. Una gammagrafía con octreótido demostró expresión moderada a alta de receptores de somatostatina, por lo que se le inició manejo con octreótido LAR mensual, logrando hasta el momento dos años de estabilidad.

Conclusiones. El octreótido constituye una alternativa de tratamiento en casos seleccionados de meduloblastoma. Se presenta revisión del tema.

\section{Angiosarcoma cardíaco primario. Reporte de dos casos}

- Lady Nataly Bedoya Trujillo, Isabel Matilde Chinchia Arias, Henry Idrobo, Fernando Huertas, Margarita Velazco, Indira Hernández, Álvaro Guerrero

Universidad Libre, Hemato Oncólogos S.A., Cali (Valle del Cauca).

Contacto: nata0916@hotmail.com

Introducción y objetivos. Es una enfermedad rara y es la neoplasia cardíaca maligna más frecuente en adultos. Se presentan dos casos de sexo femenino de angiosarcoma cardíaco primario con sus características histopatológicas e inmunohistoquímicas diagnosticados y manejados en una institución de oncología.

Materiales y métodos. La presentación inicial de las pacientes fue deterioro de la clase funcional y, en uno de los casos, metástasis a distancia. Los dos tumores se localizaron en la aurícula derecha, con un tamaño medio del tumor de $4 \mathrm{~cm}$.

Resultados. Histológicamente exhibieron la morfología de alto grado. Los tumores fueron fuertemente positivos para CD31, CD34, FLI-1 y WT-1, pero negativos para AE1/3, D2-40. Las células tumorales fueron focalmente reactivas a p53, con una alta tasa de expresión Ki-67.

Conclusiones. La resección completa del tumor fue posible en las dos pacientes por medio de cirugía cardiovascular y con quimioterapia adyuvante para mejorar la sobrevida libre de enfermedad. 